\title{
CXX. Viscosity of air in a transverse electric field
}

\section{Satyendra Ray M.Sc.}

To cite this article: Satyendra Ray M.Sc. (1922) CXX. Viscosity of air in a transverse electric field, Philosophical Magazine Series 6, 43:258, 1129-1130, DOI: 10.1080/14786442208633969

To link to this article: http://dx.doi.org/10.1080/14786442208633969

曲 Published online: 08 Apr 2009.

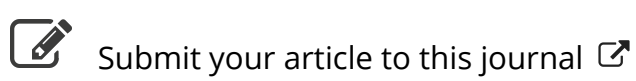

LII Article views: 1

Q View related articles $\asymp$

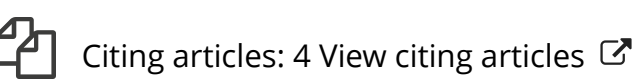


CXX. Viscosity of Air in a Transverse Electric Field. By Satyendra Ray, M.Sc. Allahalad University, Research Student, University College, London *.

UINCKE + found a change in the viscosity of liquids $\mathcal{Q}$ in an electric field. Spheres of crown and flint glass $\& c .1 \mathrm{~cm}$. in diameter were suspended by fine silk fibres attached to the arms of a balance. The logarithmic decrement was observed with and without a field due to 2000 volts, the distance between the plates being $1.5 \mathrm{~cm}$. 'The difference, which he calls electric viscosity, was 0.0398 in the case of a crown glass sphere in liquid etber when the force was transverse to the motion. Parallel to the lines of force the viscosity also increases but to a smaller extent.

Again in the case of gases the Faraday Mosotti theory of dielectrics is very well satisfied as has been tested by Boltzmann ; and if polarization physically means an orientation of molecules or a kind of tidal distortion of the molecule, $x$ change in the effective area of the cross section and a consequent change in the viscosity with the electric field might be expected.

To test whether there is any clange in the case of gases the transpiration method was employed. The usual capillary tube was replaced by two steel plates 2 feet long, 2 inches broad, and 1 inch thick. One face of each was planed and these plane faces were piaced parallel to and facing each other. The plates were separated from each other by three bits of diawn glass thread nearly $1 \mathrm{~cm}$. long on which the top plate was laid as on rollers. The sides were painted with molten resin and wax, this method being found the best to make the tube simultaneously electrically as well as pneumatically tight. The tube thus formed by parallel plates with lateral walls was connected with the usual Mariotte's bottle arrangement by means of a tin box fitted on one end of the tube with wax and resin. Paraffin oil was used in the Mariotte's bottle in the first instance, and the time of transpiration of a defiuite volume under constant pressure was noted with and without the electric field. Latterly, however, to eliminate complications arising from the use of paraffin oil, dry air was sucked through instead. For this purpose a second box was attached to the other end of the tube and connected up with a sulphuric acid wash-bottle through which the air was drawn in. The time for running out

* Communicated by Prof. A. W. Porter, F.R.S.

+ Quincke, Ann. d. Phys. u. Chem. lxii. (J897). 
water between two marks on the aspirator gauge (and afterwards for running out a definite volume of water, equal to 800 c.c. nearly), starting from the same initial conditions, was taken with and without the field.

Assuming the motion to be non-turbulent, which we reasonably can do, the viscosity must vary directly as the time of outflow. In the experiment a potential difference of 580 volts between plates $31 \mathrm{~mm}$. apart did not produce a change of even 1 sec. in $9 \mathrm{~min} .39$ sec. The potential gradient was 18000 volts per $\mathrm{cm}$. nearly, the sparking potential gradient being 31000 volts per $\mathrm{cm}$.

In setting an upper limit to a possible change in the viscosity due to the electric field, attention must be paid to the "resistance" of the other parts of the channel besides the tube. The time of flow without the field was to the time of flow with it as $4 \cdot 5: 10$ nearly. The resistance of the tube may therefore be taken to be $(10-4 \cdot 5) / 10$ of the total resistance. The present experiment may therefore be interpreted to prove the absence of any change in the viscosity of air greater than or equal in amount to 3 per cent.

I am graleful to Prof. A. W. Porter for his kind interest in the experiment and for his valuable suggestions.

list May, 1992.

\section{(YXXI. Notices respecting New Books.}

Multilinear Functions of Direction and their uses in differential geometry. By E. H. Nevtule, late Fellow of Trinity College, Cambridge, Professor of Mathematics in University College, Reading. (Cambridge: At the University Press. 8s.6 $d$. net. 1921.)

TN this small book Prof. Neville has given a systematic discus1 sion of functions of several independent directions. In the course of the development of ideas which are to some extent generalizations of the ideas of differential geometry, a number of propositions which actually occur in modern differential geometry are included. The way in which these theorems are involved in the wider theory which is here introduced, gives an insight into the co-ordinating power of the more comprehensive lines of development which the book describes.

The exposition is throughout clear and interesting. The subject matter provides one more example of the way in which mathematics covers the domains of abstract thought with ideas of ever increasing generality. 